\title{
Dendritic cells in infectious disease, hypersensitivity, and autoimmunity
}

This article was published in the following Dove Press journal:

International Journal of Interferon, Cytokine and Mediator Research

I November 2010

Number of times this article has been viewed

\author{
Brenda Gonzalez ${ }^{1,3}$ \\ Carlos Guerra' \\ Devin Morris² \\ Dennis Gray' \\ Vishwanath Venketaraman' \\ 'College of Osteopathic Medicine \\ of the Pacific, ${ }^{2}$ Graduate College \\ of Biomedical Sciences, Western \\ University of Health Sciences, \\ Pomona; ${ }^{3}$ California State Polytechnic \\ University, Pomona, CA, USA
}

Correspondence: Vishwanath

Venketaraman

Department of Basic Medical Sciences, College of Osteopathic Medicine of the Pacific, Western University of Health Sciences, 309 East Second Street,

Pomona, CA 91766, USA

Tel + I 9097063736

Fax. + I 9094695698

Email vvenketaraman@westernu.edu
Abstract: Dendritic cells are thought to direct subsequent T lymphocyte-driven adaptive immune responses (Th1/Th2) following interaction with either endogenous or exogenous antigens. These cells have an integral role in the pathogenesis of countless infectious diseases, but their role is most vividly illustrated in human immunodeficiency virus/acquired immunodeficiency syndrome and tuberculosis. Dendritic cells can arise from either myeloid or lymphoid progenitors, but the former is more common. Of particular importance are myeloid-related dendritic cells, plasmacytoid dendritic cells, infiltrating inflammatory dendritic epidermal cells, and Langerhans cells. Myeloid-related dendritic cells resemble typical monocytes, but produce cytokines that favor the cell-mediated immune response. Plasmacytoid dendritic cells resemble plasma cells, but have additional antigen-processing capabilities, and their major role is to produce interferon alpha and beta in response to viral infections. Inflammatory dendritic epidermal cells express the highest concentration of costimulatory molecules known in any cell lineage. Langerhans cells are the chief dendritic cells of the skin, and play a pivotal role in hypersensitivity and autoimmune pathologies, such as atopic dermatitis and psoriasis. Lastly, reduced glutathione has been implicated in modulation of the cytokine profiles of professional antigen-presenting cells, and holds promise as a future immunotherapeutic agent. This review discusses in detail the origin, characteristics, and overall functions of different types of dendritic cells. Furthermore, we have also highlighted the role of dendritic cells in infectious diseases and autoimmune diseases, with special reference to human immunodeficiency virus, tuberculosis, atopic dermatitis, and psoriasis.

Keywords: dendritic cell, autoimmunity, tuberculosis, HIV, innate and adaptive immunity

\section{Introduction}

Dendritic cells are professional antigen-presenting cells. Dendritic cells constitute $0.5 \%-1 \%$ of the leukocyte population in peripheral blood mononuclear cells. They are found in the majority of nonlymphoid tissues and organs, including skin, heart, liver, lung, and mucosal surfaces. ${ }^{1}$ These cells have the unique ability to link the innate and adaptive immune responses, by initiating, stimulating, and regulating $\mathrm{T}$ cell responses, including antigen-specific $\mathrm{T}$ lymphocytes, Th1/Th2 modulation, regulatory $\mathrm{T}$ cell induction, and peripheral $\mathrm{T}$ cell deletion. ${ }^{2}$

\section{Types of dendritic cells}

Dendritic cells are of four types, ie, Langerhans cells, myeloid dendritic cells, plasmacytoid dendritic cells, and infiltrating inflammatory dendritic epidermal cells. Langerhans cells are the most critical and effective dendritic cells, and have the capacity to process and present foreign antigens to T cells. These resident cells are located in the 
epidermal and dermal layers of the skin. Classical Langerhans cells are characterized ultrastructurally by a clear cytoplasm, a lobulated nucleus, and a lack of desmosomes, melanosomes, or Merkel cell granules, and, most importantly, by the presence of highly specific tennis racket-shaped cytoplasmic Birbeck granules. Unlike inflammatory dendritic epidermal cells, they express CD1a and CD207 on their surface. Both cell types (Langerhans cells and inflammatory dendritic epidermal cells) have been shown to express high levels of Fc epsilon receptor I (FceRI), the high-affinity receptor for the constant region of the IgE antibody, on their surface. The ultrastructure of inflammatory dendritic epidermal cells resembles that of Langerhans cells because of a clear cytoplasm, lobulated nucleus, and lack of desmosomes/melanosomes or Merkel cell granules. However, unlike classical Langerhans cells, inflammatory dendritic epidermal cells do not contain any Birbeck granules

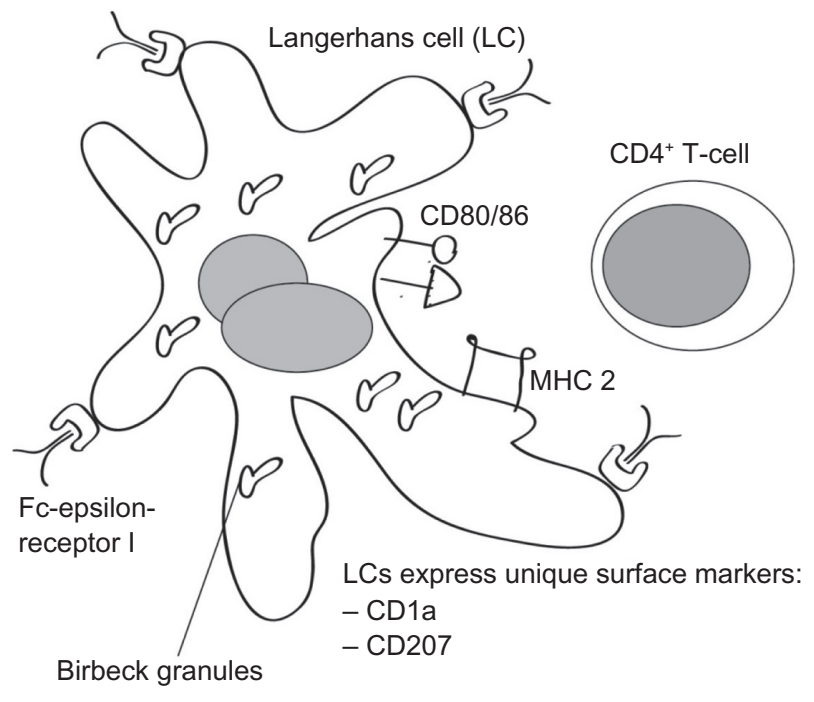

Inflammatory dendritic epidermal cell (IDEC)

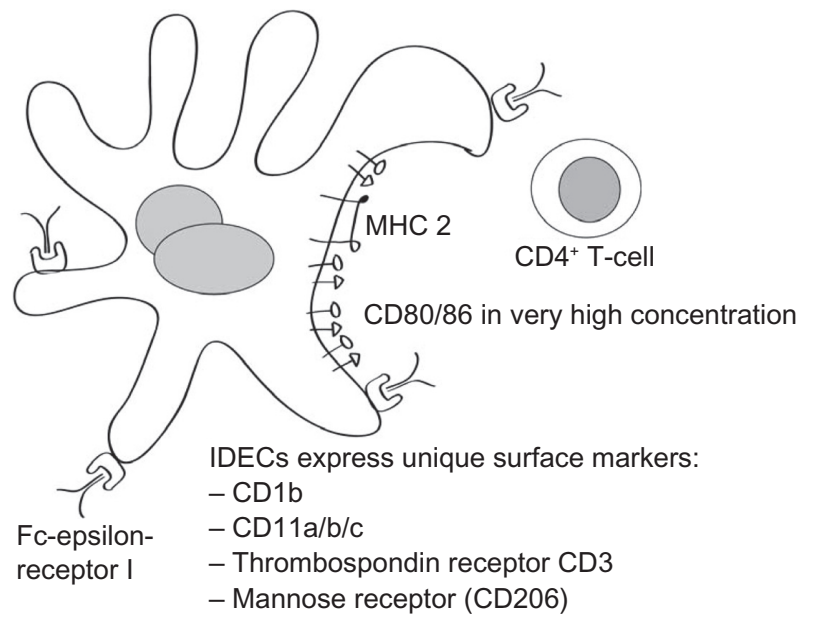

Figure I Langerhans cells compared with inflammatory dendritic epidermal cells. Both cell types express certain unique surface markers; however, Birbeck granules are the major distinguishing feature.
(Figure 1). Moreover, inflammatory dendritic epidermal cells additionally express CD1b, CD11a, CD11b, and CD11c, the thrombospondin receptor (CD36), and the mannose receptor (CD206), which is known to be involved in the uptake of bacterial components. Inflammatory dendritic epidermal cells have been shown to express even more costimulatory molecules, such as CD80 and CD86, than Langerhans cells (Figure 1).

\section{Monocytoid and plasmacytoid dendritic cells}

Dendritic cells are a heterogeneous population of immune cells which are thought to exert different functions, depending on their origin, state of activation, and location. While dendritic cells can arise from either myeloid or lymphocytic progenitors, dendritic cells arising from the myeloid lineage are much more common. Specifically, it has been shown that myeloid-related dendritic cells $\left(\mathrm{CD} 11 \mathrm{c}^{+} / \mathrm{CD} 33^{+} / \mathrm{CD} 13^{+}\right)$ and plasmacytoid dendritic cells (CD11 $\left.\mathrm{c}^{-} / \mathrm{IL} \mathrm{T}^{+} / \mathrm{ILT}^{-}\right)$are the most common variants. Monocyte-derived dendritic cells arise from monocytes that have migrated from the bloodstream into peripheral tissues and maintain migratory ability within these tissues, giving myeloid dendritic cells the capacity to reach mucosal-associated lymphoid tissues. Plasmacytoid dendritic cells, previously known as T-associated plasma cells, ${ }^{3}$ are localized in the fetal liver, thymus, and bone marrow, which suggests that plasmacytoid dendritic cells develop from hematopoietic stem cells in primary lymphoid tissues. ${ }^{4,5}$ Plasmacytoid dendritic cells arise from plasmacytoid cells that play an important role in innate immunity and can serve as antigen-presenting cells. These types of dendritic cells contain internal tolllike receptors (TLR), ie, TLR-7 and TLR-9, which bind to single-stranded RNA genomes. However, TLR-9 binds to unmethylated cytosine in dinucleotide $\mathrm{CpG}$ islands of DNA in pathogens. TLR7 and TLR 9 are exclusive to plasmacytoid dendritic cells, which are known to secrete high concentrations of interferon (IFN)- $\alpha$ in response to viral infection.

With localization comes functionality, because plasmacytoid dendritic cells secrete IFN- $\alpha$ in response to cellular sensors that promptly detect the presence of DNA and RNA viruses. ${ }^{3}$ These sensors come from the expression ofTLR-7 and TLR-9, as mentioned earlier, and are located in the endosomal compartment of plasmacytoid dendritic cells, and not on the cell surface. TLR-9 expression accounts for the plasmacytoid dendritic cell response to $\mathrm{CpG}$ oligonucleotides, which mimic bacterial DNA. ${ }^{3}$ Plasmacytoid dendritic cells are able to migrate independent of inflammatory stimuli and foreign antigens. In addition, recent evidence suggests that plasmacytoid 
dendritic cells produce high levels of IFN- $\alpha$, allowing for a range of immunoregulatory effects. ${ }^{6}$ Mature plasmacytoid dendritic cells express major histocompatibility complex (MHC) Class I and II molecules, and are further upregulated following activation. Plasmacytoid dendritic cells are recruited to nonlymphoid tissue in inflammatory pathologies, including rheumatoid arthritis, cutaneous lupus erythematosus, and allergic reaction sites. Plasmacytoid dendritic cells have also been shown to accumulate in human ovarian carcinoma. ${ }^{\text {? }}$

\section{Differences between monocytoid and plasmacytoid dendritic cells}

There are several features that distinguish myeloid from plasmacytoid dendritic cells. Myeloid dendritic cells express c-kit markers and are associated with monocytes and macrophages of the cell-mediated immune response. Characteristically, these cells release large amounts of interleukin (IL)-12 in response to bacterial antigen recognition by TLR, most notably TLR-2, 3, and 4. On the other hand, plasmacytoid dendritic cells (CD11c-/MHC Class II+), more closely resemble plasma cells, with additional endocytic and antigenic processing abilities. These cells express the BDCA-2 marker (a unique Type II C-type lectin that has shown ability to inhibit IFN- $\alpha / \beta$ induction) together with the IL-7R (Table 1$)$. It seems that a primary role for plasmacytoid dendritic cells may be the production of IFN- $\alpha$ in response to viral infections. ${ }^{2}$ Interestingly, because plasmacytoid dendritic cells express IL-3 $\alpha \mathrm{R}$ (CD123) and CD62L (L-selectin), this cell lineage is

Table I Differences between monocytoid and plasmacytoid dendritic cells

\begin{tabular}{|c|c|c|c|}
\hline DC subset & $\begin{array}{l}\text { MDC } \\
\left(\mathrm{CDI} \mathrm{Ic}^{+} / \mathrm{CD}_{3} 3^{+} /\right. \\
\left.\mathrm{CDI}^{+}\right)\end{array}$ & $\begin{array}{l}\text { PDC } \\
(\text { CDI I c-/IL3T }+/ \\
\text { ILTI') }\end{array}$ & Both \\
\hline Chemokine & CCRI & CXCR3 & CCR2 \\
\hline receptor & CCR3 & & CCR4 \\
\hline \multirow[t]{4}{*}{ expression } & CCR6 & & CCR5 \\
\hline & CXCRI & & CCR7 \\
\hline & & & CXCR2 \\
\hline & & & CXCR4 \\
\hline DC surface & MMR & MHC I & CDIIa \\
\hline antigen & c-kit & MHC II & II-7R \\
\hline \multirow[t]{5}{*}{ receptor } & CD46 & CD40 & BDCA-2 \\
\hline & CDIIb & CD80 & CDI23 \\
\hline & CDIIc & CD86 & CD622 \\
\hline & CD32 & & CLA4 \\
\hline & CD64 & & CD34 \\
\hline Toll-like & TLR-2 & TLR-7 & \\
\hline \multirow[t]{2}{*}{ receptor } & TLR-3 & TLR-8 & \\
\hline & TLR-4 & TLR-9 & \\
\hline Cytokine & IL-2 & IFN- $\alpha$ & \\
\hline production & IL-I5 & & \\
\hline
\end{tabular}

sensitive to maturation when stimulated with IL-3 or CD40, respectively. ${ }^{4}$ Plasmacytoid dendritic cells also seem to be longer-lived than other subsets of dendritic cells because they are constantly circulating throughout the blood and lymphoid organs. The ability of plasmacytoid dendritic cells to circulate in such a manner has been attributed to their expression of CD62L and CLA-4, which are ligands for CD62E and CD62P, and respectively bind $\mathrm{E}$ and $\mathrm{P}$ selectins on endothelial vessels in peripheral tissues. ${ }^{2}$

Along with surface markers that distinguish dendritic cells, there are chemokine receptors present on dendritic cells that correspond to the level of maturation of the lineage. Immature dendritic cells express CCR2, an important receptor to translocate dendritic cells to T-cell-rich regions of lymphoid tissue and CXCR4 and CCR4, which are receptors for CXCL12 (SDF-1a) and CCL22 (myeloid dendritic cells) or CCL12 (thymus and activation-regulated cells), respectively. They also express the inflammationinduced chemokines, CCR1, CCR3, CCR5, and CXCR1 (Table 1). CCR6 positions dendritic cells on epithelial surfaces, and CCR7 aids migration to T cell areas of lymph nodes. Upon activation, immature dendritic cells migrate to draining lymph nodes and develop into mature effector dendritic cells. Dendritic cells differ in function due to locality in the human body, eg, skin, gastrointestinal tract, and lungs (Table 1).

\section{Functions of dendritic cells}

The final event leading to activation of the adaptive immune response is activation of helper T cells, which greatly amplify cytokine cascades, and are thought to have the dominant influence on progression to either a cell-mediated (Th1) or antibody-mediated (Th2) immune response to pathogens. Dendritic cells are the most effective antigen-presenting cells for inducing maturation of naïve $\mathrm{T}$ cells because they express the highest concentration of MHC Class II molecules on their surface (Figure 2). MHC Class I peptides are categorized by endogenous antigenic processing via the cytosolic pathway, and MHC Class II peptides are categorized by exogenous antigenic processes via the endocytic pathway. MHC Class II molecules engage specific $T$ cell receptors. However, this alone is not enough to cause activation and maturation of T cells; the antigen-presenting cell must also express costimulatory molecules, while the $\mathrm{T}$ cell must express activation receptors for the costimulators. Costimulatory molecules on dendritic cells, such as CD80, CD86 (Figure 2), and CD40, play a major role in the activation of naïve $\mathrm{T}$ lymphocytes, by engaging the respective receptors and ligands, ie, the 

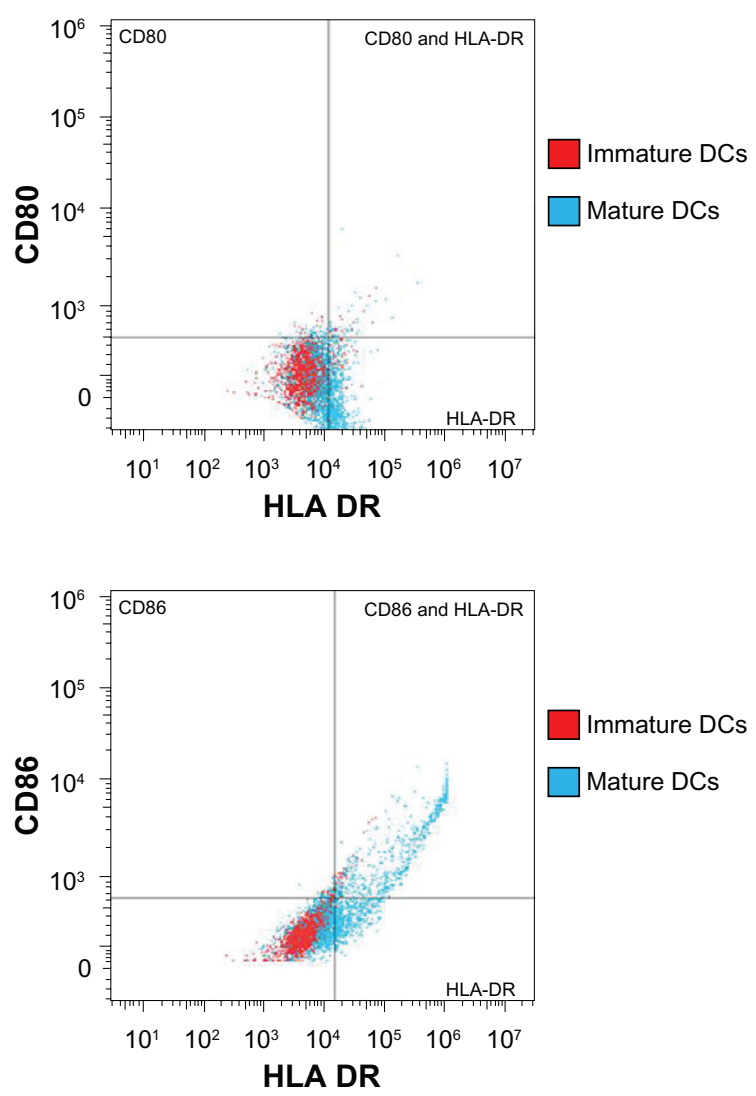

Figure 2 Flow cytometry showing expression of CD80 (B7.I) and CD86 (B7.2) costimulatory molecules on both mature and immature dendritic cells expressing human leukocyte antigen-DR, a major histocompatibility complex Class II molecule.

second signal. If either end of this so-called second signal is missing, typically the $\mathrm{T}$ cell is anergized, making it unreactive to activation.

The ability of dendritic cells to induce $\mathrm{CD} 4^{+} \mathrm{T}$ cells to differentiate into the Th1 or Th2 phenotype is dependent on IL-12 production. IL-12, a heterodimeric $70 \mathrm{kD}$ cytokine, is mainly produced by dendritic cells in response to bacterial products, such as lipopolysaccharide, or to intracellular pathogens. IL-12 was first discovered because of its ability to induce IFN- $\gamma$ production and cell proliferation (Figure 3). It is now known that IL-12 also plays a key role in the development of Th1 responses, leading to IFN- $\gamma$ and IL-2 production (Figure 4). These cytokines can, in turn, promote $\mathrm{T}$ cell responses and macrophage activation. ${ }^{8} \mathrm{IFN}-\gamma$ in synergy with other cytokines, such as TNF- $\alpha$, activates macrophages to control intracellular infection. ${ }^{8,9}$

There are several protocols for in vitro generation of dendritic cells. For plasmacytoid dendritic cells, in vitro generation of CD34+/CD45RA-/IL-3Ra- plasmacytoid dendritic cells can be cultured with FMS-like tyrosine kinase 3 ligand. Maturation can be induced with IL-3 and CD40 by IL-3 and TNF- $\alpha$. In vivo activation and maturation of plasmacytoid

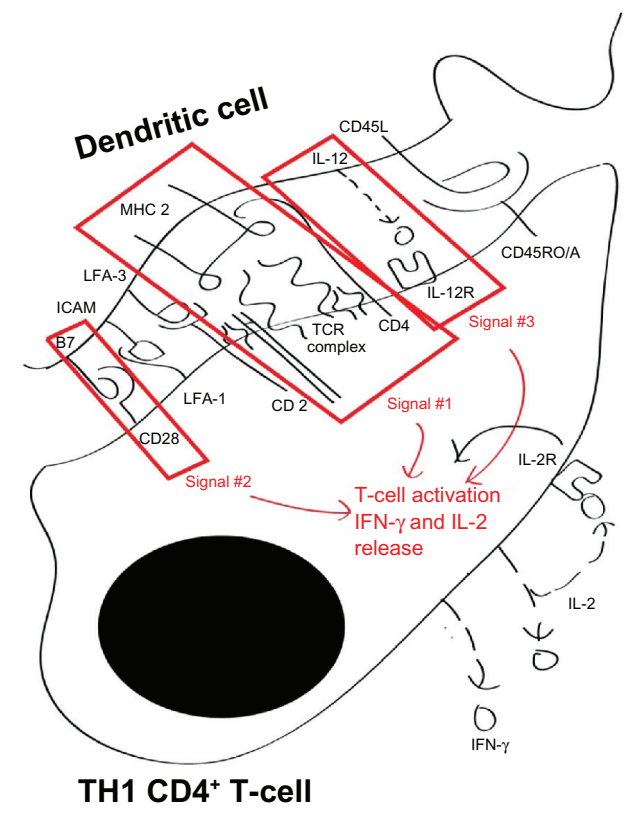

Figure 3 Activation of $\mathrm{CD} 4^{+} \mathrm{T}$ lymphocytes of the ThI subset occurs in a stepwise fashion requiring three signals. The first signal involves major histocompatibility complex Class II engaging the $T$ cell receptor complex. The second signal is achieved by interaction of costimulator molecules on the dendritic cells with their respective receptors on the T cell (B7.I and B7.2 are analogous to CD80 and CD86, respectively). Lastly, IL- 12 must bind the IL- 12 receptor on the $T$ cell for complete activation. Following activation, CD45A is no longer expressed by the cell, and is replaced by $\mathrm{CD} 45 \mathrm{O}$, hence $\mathrm{CD} 45 \mathrm{O}^{+} \mathrm{T}$ cells are considered "activated". These events culminate in release of interferon-gamma (IFN- $\gamma$ ) and IL-2. IL-2 stimulates an autoreceptor on the $\mathrm{T}$ cell to maintain the active state. Both these cytokines, IFN- $\gamma$ and IL-2, stimulate the cell-mediated arm of the immune response, notably activation of macrophages, enabling destruction of intracellular bacteria.

dendritic cells depends on which TLR is engaged. TLR3 is specific for viral RNA and poly IC, while TLR $2 / 4$ is more specific for mycobacterial extracts and antibody-opsonized foreign bodies bound to constant region antibody fragment receptors. Dendritic cell maturation is also induced by proin-

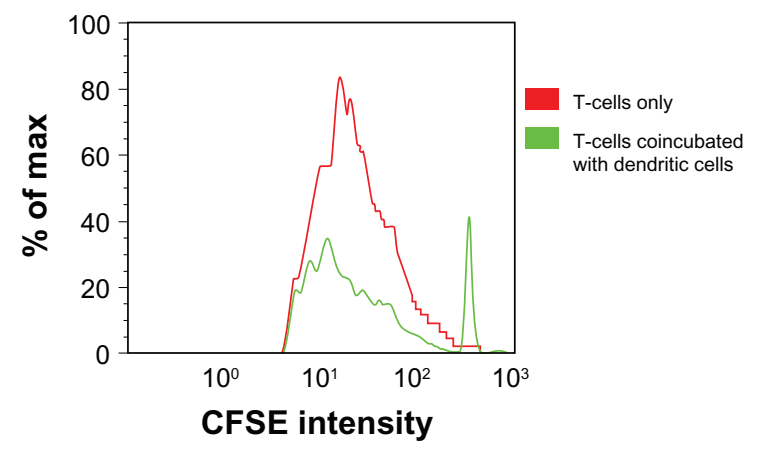

Figure $4 \mathrm{~T}$ cell proliferation in response to antigens presented by dendritic cells. $T$ cells were stained with carboxyfluorescein diacetate succinimidyl ester (CFSE), a fluorescein molecule, to assess T cell proliferation upon coincubation with dendritic cells infected with Mycobacterium tuberculosis. CFSE contains a succinimidyl ester functional group and two acetate moieties, it diffuses into the cell and intracellular esterases cleave the acetate groups, converting them into a fluorescent membrane impermanent dye; during each cell division, the fluorescent CFSE is partitioned equally between daughter cells. T cell proliferation is indicative of IL-2 production by $\mathrm{T}$ cells and a ThI response. 
flammatory stimuli, including tumor necrosis factor alpha, IFN $\gamma$, IL-1b, and prostaglandins. ${ }^{7}$

\section{Glutathione and regulation of dendritic cell function}

Glutathione is a tripeptide that, in its reduced form, protects cells against oxidizing agents, free radicals, and reactive oxygen intermediates. In addition to its antioxidant role, glutathione plays a vital role in maintenance of cell viability and regulating immune cell functions. ${ }^{10}$ Glutathione levels in antigen-presenting cells play a central role in determining which of the cytokine (Th1 or Th2) patterns predominate in the immune response. ${ }^{10}$ It has been shown that depletion of glutathione in antigen-presenting cells inhibits Th1-associated cytokine production and/or favors Th2-associated responses. ${ }^{10}$

\section{Dendritic cells and infectious disease}

Infectious diseases have been the leading cause of death in underdeveloped countries around the world for many decades. Diseases such as acquired immunodeficiency syndrome (AIDS), tuberculosis, and acute lower respiratory tract infection are some of the common infectious diseases that have now become drug-resistant within the past decade and are becoming an emerging threat to the human race, especially in immune-compromised populations. The kinds of re-emerging diseases that have become resistant include methicillin-resistant Staphylococcus aureus and multidrug-resistant tuberculosis. It is now common to see strains of Mycobacterium tuberculosis that are extremely drug-resistant, in which the pathogen has become completely resistant to every type of medication available. How can these threats have emerged from their once isolated infection region to become a global pandemic? Increase in population, human travel from country to country, ecologic changes, poverty, and food processing methods can be factors for emergence of these infectious diseases and their travel to unexposed populations of the world. It is uncertain whether advancements in cellular and molecular biology, and the ongoing pursuit to control the inevitable infectious disease pandemics due to human factors such as an increase in antibiotic consumption, food treatment, and processing will be enough to combat this never-ending threat to human society.

The importance of dendritic cells in infectious diseases is being elucidated in great detail to correlate the proper activation needed to elicit a Th1 or Th2 response to favor a pathogenic termination. With this in mind, we take a step back and discuss ongoing research of the effectiveness of dendritic cells in combating infectious diseases.

\section{Dendritic cells and HIV}

As aforementioned, dendritic cells are the first lines of defense against infection by pathogens, and serve as an invaluable link between the innate and adaptive immune responses. Consequently, their role in the progression of HIV infection has been widely studied. Many studies have shown that the normal functions of dendritic cells are hijacked by HIV, and used as a reservoir to facilitate the dissemination of the virus, while at the same time limiting the ability of the immune system to destroy it.

During viral infections, natural killer cells induce apoptosis of infected immature dendritic cells through direct cell-to-cell interaction, ${ }^{11}$ while at the same time promote the maturation of immature dendritic cells into mature dendritic cells and their migration to lymph nodes through the secretion of an alarmin called high mobility group box 1 (HMBG1) protein. This process prevents further spread of the virus, while at the same time mobilizes the adaptive immune response by facilitating antigen presentation in the lymph nodes. However, the HIV virus has hijacked this process, as noted by a recent study demonstrating that, in dendritic cells infected by the HIV virus, HMGB1 causes increased expression of cellular-flice-like inhibitory protein (c-FLIP) and the cellular inhibitor of apoptosis-2 which inhibits apoptotic killing of dendritic cells by natural killer cells. ${ }^{12}$

Another study has demonstrated that dendritic cell infection by HIV leads to increased secretion of IL-10, which also confers resistance to killing by natural killer cells to immature dendritic cells, while at the same time increases the susceptibility of mature dendritic cells to apoptotic killing by natural killer cells. ${ }^{13}$ This acquired resistance to natural killer cell cytotoxicity allows infected immature dendritic cells to travel to the lymph nodes where they disseminate the virus to $\mathrm{CD} 4^{+} \mathrm{T}$ cells.

There is further evidence that HIV uses dendritic cells to facilitate travel to the lymph nodes where it can be transmitted to awaiting $\mathrm{CD}^{+} \mathrm{T}$ cells. A recent study has shown that, in patients infected with the HIV-1 virus, plasmacytoid dendritic cells demonstrate increased expression of CCR7 and CD62 L, a lymph node homing marker, and cellular adhesion molecule, respectively. These plasmacytoid dendritic cells have an increased capacity for migration to the lymph nodes, and consequently accumulate at these sites. These accumulated plasmacytoid dendritic cells secrete greatly increased levels 
of IFN- $\alpha$, a cytokine which activates natural killer cells and macrophages, as well as recruiting activated T cells, and promotes differentiation of CD4 ${ }^{+} \mathrm{T}$ cells into effector cells. ${ }^{14,15}$

An in vitro study has also demonstrated that inactivated HIV is capable of inducing the production of IFN- $\alpha$ by plasmacytoid dendritic cells. ${ }^{16}$ This increased IFN- $\alpha$ production can potentially lead to the recruitment of large numbers of $\mathrm{CD}^{+}$ T-cells, facilitating their infection and subsequent depletion by the HIV virus. This dissemination of the virus occurs due to HIV exploitation of a dendritic cell-specific surface receptor called dendritic cell-specific intracellular adhesion molecule (ICAM)-3 grabbing nonintegrin (DC-SIGN). DC-SIGN is an ICAM-3 receptor specific to dendritic cells, and is highly expressed in immature dendritic cells. The HIV glycoprotein gp120 has a high binding affinity for DC-SIGN, which allows the virus to gain entry into the cell. ${ }^{17}$ Upon migration of dendritic cells to the lymph node, DC-SIGN plays an integral role in the formation of an "infectious synapse" which facilitates the transfer of HIV from dendritic cells to naïve T cells. ${ }^{18}$ Furthermore, chronically elevated levels of IFN- $\alpha$ can contribute to HIV-associated wasting syndrome, which is seen in many HIV patients.

In addition to commandeering dendritic cell mechanisms of interaction with T cells, HIV also interferes with the ability of dendritic cells to prime naïve $\mathrm{T}$ cells. This priming normally occurs when dendritic cells present viral antigens complexed with MHC Class I or MHC Class II molecules to $\mathrm{CD}^{+}$or $\mathrm{CD}^{+} \mathrm{T}$ cells, respectively. However, naïve $\mathrm{CD} 4^{+} \mathrm{T}$ cells primed by HIV-infected dendritic cells show increased expression of CTLA-4, PD-1, TRAIL, and Foxp3 molecules, which are known to inhibit $\mathrm{T}$ cell proliferation and lead to anergy of HIV antigen-reactive T cells. ${ }^{19}$

HIV infection also leads to a reduction in the expression of CD1d molecules on the surface of dendritic cells. CD1d serves as an antigen presentation molecule for lipid antigens. Presentation of lipid antigens via CD1d molecules activates natural killer T cells. These natural killer T cells serve as an early link between the innate and adaptive immune response, secreting cytokines leading to the Th1 or Th2 response. Natural killer T cells also have the capacity to regulate other cell types, such as macrophages and dendritic cells. Thus, by interfering with the surface expression of CD1d molecules of dendritic cells, HIV can disrupt many aspects of the immune response. ${ }^{20}$

As discussed previously, TLR-7 and TLR-9 present on plasmacytoid dendritic cells are used in responding to viral infections associated with ssRNA, such as in the HIV-1 genome. Plasmacytoid dendritic cells are believed to play an important role in recognition of HIV-1 because they secrete high amounts of IFN- $\alpha$ for inducing antiviral states in nucleated cells, as well as increasing MHC Class I expression.

\section{Glutathione and HIV infection}

Findings from our research laboratory, as well as by other groups, confirm that intracellular levels of glutathione are decreased in patients with AIDS, ${ }^{22}$ in whom the risk of tuberculosis is many times that of healthy individuals. ${ }^{22-24}$ The factors responsible for low glutathione in HIV infection are poorly understood. Infection with HIV is believed to trigger a range of metabolic changes, in addition to the progressive deficits in cellular immunity and increased susceptibility to opportunistic infections that are its clinical hallmarks, and the progression to AIDS. ${ }^{22,23}$ The decreased glutathione content in immune cells of HIV-positive individuals is at least in part attributed to the decrease in plasma cysteine and increased plasma glutamate (an inhibitor of cysteine permeation via the Xc-transport system), as observed during early infection. The decreased intracellular glutathione and plasma cysteine observed in HIV patients may also be due to chronic oxidative stress, and this may lead to progression of the disease. The decreased availability of cysteine can be overcome to some extent by the cysteine precursor, $n$-acetylcysteine (NAC) ${ }^{25}$ Herzenberg et al found that NAC treatment improves the clinical situation and delays disease progression. ${ }^{22}$ This study has shown that long-term NAC administration to AIDS patients improves their hematologic profile, glutathione content, and life expectancy. ${ }^{22-24}$ We therefore speculate that dendritic cell functions will be compromised in individuals who are infected with HIV and this is due to decreased levels of intracellular glutathione.

\section{Tuberculosis}

M. tuberculosis is the oldest infection to plague the human species and continues to be a major public health concern today. One third of the world's population is infected with M. tuberculosis. Of the two billion people infected, $90 \%$ have what is known as latent tuberculosis infection. ${ }^{26}$ Individuals with latent tuberculosis infection are asymptomatic, and $10 \%$ of these individuals will develop reactivation, culminating in an active tuberculosis infection secondary to a compromised immune system caused by stress, age, malnutrition, therapy with corticosteroids or other immunosuppressive drugs, malignancy, end-stage renal disease, and, most importantly, coinfection with HIV. Moreover, there is a rise in multidrug-resistant and extremely drug-resistant isolates, particularly in Africa and Russia. ${ }^{26}$ 


\section{Dendritic cells and M. tuberculosis}

M. tuberculosis is an aerobic, acid-fast, slow-growing, nonmotile bacillus, and a facultative and successful intracellular pathogen. The bacteria are transferred from one infected individual to another by coughing droplet nuclei that can contain as few as $1-3$ bacilli. ${ }^{27}$ The bacteria colonize the middle lung zone, where airflow is greatest to maximize aerobic respiration. Alveolar macrophages (dust cells), along with dendritic cells, phagocytose the bacteria and initiate an innate as well as an adaptive immune response. Combined efforts by macrophages and dendritic cells establish protective immunity in $90 \%$ of infected individuals. In macrophages, M. tuberculosis can attenuate antigen presentation via the MHC II complex, as well as take control of phagosomes, thereby preventing formation of phagolysosomal fusion. ${ }^{28}$ However, M. tuberculosis is not completely eradicated, making the chance of a reactivation possible. There are several defensive mechanisms that M. tuberculosis has developed to evade the immune cell response. Upon antigen uptake, dendritic cells reduce their endocytic/phagocytic capabilities and begin a process of maturation. Maturation includes expression of MHC Class II molecules, along with costimulatory molecules, including CD80, CD86, and CD40, essential in antigen presentation to T lymphocytes. ${ }^{1}$ Additionally, dendritic cells express chemokines CCR6 and CCR7, which are essential in migration to draining lymph nodes. Lipoarabinomannan and cell wall-associated alpha-glucan, both mycobacterium cell wall components, are known to suppress secretion of IL-12 and negatively affect dendritic cell function. ${ }^{28}$ There needs to be further research to elucidate the mechanism allowing $M$. tuberculosis to attenuate dendritic cell responses, because such insight would potentially lead to treatment without eliciting immunopathology.

\section{Dendritic cells, hypersensitivity, and autoimmunity}

Dendritic cells appear to have a substantial role in autoimmune reactions involving the skin. The most important role of the skin may be the protection against environmental threats, such as microbes, ultraviolet light, or chemicals. Skin is highly impermeable and prevents microorganisms from gaining access to internal organs. The outer layer of dead cells on the skin is sloughed off, eliminating organisms that are bound to the skin. Skin contains sebaceous glands that secrete sebum which maintains a low $\mathrm{pH}$, thereby preventing colonization by bacteria. Skin also secretes lysozymes and antimicrobial peptides including cathelicidins and defensins. If the epidermal barrier is disrupted, pathogens as well as allergens can make contact with the resident innate immune cells in the skin. Dendritic cells are optimally positioned within the dermis, and detect any skin invading pathogen and allergen. As mentioned earlier, the major function of dendritic cells is the initiation of adaptive immune responses, specifically the presentation of microbial antigens to $\mathrm{T}$ lymphocytes and the modulation of $\mathrm{T}$ lymphocyte differentiation and activation. Current areas of interest involve the contribution of innate mechanisms (eg, TLRs/ pattern recognition receptors) to the development of dendritic cell-mediated sensitization, activation, and specific subset proliferation. Of particular interest is the role of dendritic cells in atopic dermatitis and psoriasis.

\section{Skin and its layers}

The skin is composed of an outer layer (epidermis) and an inner layer (dermis). The epidermis is made of four layers. The other layers of skin, in descending order, comprise the stratum corneum, stratum lucidum, stratum granulosum, stratum spinosum, and stratum basale. The outermost layer of epidermis, the stratum corneum, is made of denucleated squamous cells originating from keratinocytes in the stratum basale. The dermis comprises blood vessels, nerves, and collagen and elastin fibers. Dendritic cells, keratinocytes, and mast cells are resident cells that contribute in many ways to optimal innate defense mechanisms of the skin.

In the skin, dendritic cell subsets are classified as Langerhans cells, resident dermal myeloid dendritic cells, plasmacytoid dendritic cells, and myeloid dermal inflammatory dendritic cells. Langerhans cells, as well as dermal dendritic cells, can take up antigen, process it into fragments, and migrate to regional lymph nodes where they present the antigen to cells of the adaptive immune system. The antigens processed by dendritic cells can either be of microbial origin, leading to antimicrobial adaptive immune responses, or it can be an allergen, which may lead to immediate Type 1 or delayed Type 4 hypersensitivity reactions.

\section{Atopic dermatitis}

Atopic dermatitis is one of the most common chronic inflammatory skin diseases, affecting $10 \%-20 \%$ of children and $1 \%-3 \%$ of adults in industrialized countries. Atopic dermatitis occurs slightly more frequently in women than in men by a ratio of approximately $1.5: 1.3 .^{29}$ The majority of cases (at least $60 \%$ ) arise within the first year of life. The remainder appears in two peaks, ie, age 2-12 years and from puberty into adulthood. ${ }^{29}$ The etiology of atopic dermatitis involves a complex interplay between environmental triggers and genetic factors, including altered innate and adaptive immune responses. The 
etiology and clinical course of atopic dermatitis is not well understood. However, there appears to be a multifaceted interaction between skin barrier dysfunction, hyperreactivity of distinct immune cells, and elevated exposure to systemic allergens over the course of one's lifetime.

The histologic features of atopic dermatitis depend on the duration of the disease. Acute lesions are characterized by marked intercellular edema of the epidermis and a sparse epidermal infiltrate consisting primarily of $\mathrm{T}$ lymphocytes. Marked perivascular inflammatory cell infiltrates, consisting predominantly of T lymphocytes and occasional monocyte/ macrophages, are seen in the dermis. An increased number of IgE-bearing Langerhans cells in the epidermis are present, and macrophages dominate the dermal mononuclear cell infiltrate. The number of mast cells are increased but are generally fully granulated. Increased numbers of eosinophils are observed in chronic atopic dermatitis skin lesions, although not seen in acute lesions. Eosinophil cationic protein is elevated in atopic dermatitis sera and correlates with disease severity, providing evidence for eosinophil activation and involvement in atopic dermatitis. Atopic dermatitis patients display apparently defective innate immune responses, which leads to increased susceptibility to viral, bacterial, and fungal infections. An example of this is the almost 90\% rate of colonization of the skin with Staphylococcus aureus in patients with atopic dermatitis, compared with only $5 \%$ colonization in healthy people. ${ }^{30}$

Many clinical features of atopic dermatitis are a direct consequence of the atopic patient's tendency toward Th2skewed acquired immune responses. Although the Th1/Th2 paradigm model, with $\mathrm{Th} 2$ responses predominating in atopic dermatitis, has made a major contribution to our understanding of immunologic processes, the actual mechanisms involved may be more complex. In atopic dermatitis, patients appear to develop a biphasic helper T cell pattern, with Th2 cytokine predominance seen early in the acute stage, but then a switch to a more Th1-like profile characteristic of the chronic pathology, with higher levels of IFN- $\gamma$. Compared with normal skin, both acute and chronic atopic dermatitis lesions contain significantly more cells expressing messenger RNA (mRNA) for IL-4, IL-5, and IL-13, but the expression of IFN- $\gamma$ is similar to that seen in normal skin. However, compared with acute lesions, chronic lesions are associated with significantly fewer IL-4 and IL-13 mRNA-expressing cells, significantly more IL-5 and IL-12 mRNA-expressing cells, and significantly more antieosinophil cationic protein antibody. These data suggest that maintenance of chronic inflammation in atopic dermatitis lesions may be associated with the predominance of IL-5 and IL-12 expression and eosinophil infiltration. IL-12 could mediate a switch from Th2 predominance during the acute phase to greater Th1 involvement during the chronic phase.

In atopic dermatitis, as in other inflammatory skin diseases, the localization of the pathogenic $\mathrm{T}$ cells to the skin is likely to be regulated by the expression of a specific skin-homing receptor on these cells. This receptor, or cell-adhesion molecule, known as cutaneous lymphocyteassociated antigen, interacts with vascular endothelial cell surface antigens to direct circulating $\mathrm{T}$ lymphocytes to reactive skin sites, and defines a subset of circulating memory $\mathrm{T}$ cells. Cutaneous lymphocyte-associated antigen interacts with the vascular receptor, E-selectin, on inflamed dermal postcapillary venules, which forms the basis of the selective and immediate recruitment of cutaneous lymphocyteassociated antigen-positive $\mathrm{T}$ cells into the skin on exposure to foreign pathogens or allergens. An increased number of activated cutaneous lymphocyte-associated antigen-positive $T$ cells have been reported in the blood of patients with atopic dermatitis. ${ }^{31}$ Other important adhesion molecules involved in the homing process to inflammatory sites are $\beta-6$ integrin, vascular cell adhesion molecule (VCAM)-1, ICAM-1, and IL-8, all of which can be found in higher levels in the peripheral blood of patients with atopic dermatitis. ${ }^{32}$ It has been reported that in normal human skin, $\beta-6$ integrin is weakly expressed by blood vessels and by basal epithelial cells of the epidermis. However, in acute and chronic lesional skin of patients with atopic dermatitis, dramatic upregulation of $\beta-6$ integrin expression is observed on endothelial cells and in the epidermis. ${ }^{33}$

Two different types of epidermal dendritic cell populations have been described in lesions of atopic dermatitis, ie, Langerhans cells and inflammatory dendritic epidermal cells. FceRI levels on Langerhans cells in the lesional skin of patients with atopic dermatitis are significantly higher than in uninvolved areas, although Langerhans cells at nonlesional sites still bear higher receptor numbers than in skin of normal controls. ${ }^{34}$ Atopic dermatitis lesions also contain another distinct population of $\mathrm{CD} \mathrm{a}^{+}$dendritic cells, called inflammatory dendritic epidermal cells, which are not found in normal skin. Like Langerhans cells, inflammatory dendritic epidermal cells express FceRI on their surface and likely play an important role in allergen presentation to $\mathrm{T}$ cells in atopic dermatitis, and FceRI expression may be even greater in inflammatory dendritic epidermal cells than in classic Langerhans cells. It is thought that the high affinity receptor for immunoglobulin E-expressing Langerhans 
cells in the epidermis play a crucial role in the early events of atopic dermatitis. Because of the reduced skin barrier function in patients with atopic dermatitis, allergens could readily penetrate into the epidermis and be taken up by the FceRI-bound IgE molecules on epidermal Langerhans cells. In this way, Langerhans cells could potentially respond to numerous allergens by binding $\mathrm{IgE}$ molecules with various specificities, leading to very efficient processing and targeting of antigen to MHC Class II-rich compartments. The presence of high affinity FceRI, therefore, enables Langerhans cells to capture and internalize the antigen before antigen is presented to $\mathrm{T}$ cells, in turn leading to optimal antigen presentation to $\mathrm{T}$ cells as a first-line mechanism for antigen recognition. After the initial contact of Langerhans cells with antigens in skin, Langerhans cells migrate to the draining lymph nodes. In addition to migration, FceRI activation of Langerhans cells leads to the release of chemokines, such as monocyte chemoattractant protein 1, IL-16, thymus and activation-regulated chemokine, and macrophage-derived chemokine, which might be responsible for the recruitment of other proinflammatory cells into the skin. It is in the lymph node that polarization of naive T cells into Th1 versus Th2 subsets occurs under the direction of dendritic cells, while Langerhans cells activated by FceRI drive naive T cells into IL-producing T cells of the Th2 type. It has been shown that in lesional skin of atopic dermatitis, there is a significant increase in the number of dendritic cell-shaped CD1a+ cells within the epidermal layer compared with controls, reflecting the augmented antigen-presenting function of these cells. ${ }^{34}$

The increased expression of CD86 in atopic dermatitis lesional skin has been demonstrated to have a functional relevance, thus supporting a role for inflammatory dendritic epidermal cells in the presentation of antigens in atopic dermatitis skin..$^{32}$ In line with these data, expression of FceRI is highly upregulated on $\mathrm{CD} \mathrm{a}^{+}$cells from patients with atopic dermatitis, compared with levels in normal and nonlesional skin, and expression is significantly correlated with serum IgE levels. ${ }^{35}$ Increased numbers of CD1a+ dendritic cells and upregulated expression of FceRI should increase the capacity for antigen/allergen capture and presentation of antigen/ allergen to $\mathrm{CD} 4^{+} \mathrm{T}$ lymphocytes. IgE-bearing $\mathrm{CD}^{2} \mathrm{a}^{+}$dendritic cells that have captured antigen/allergen may also activate memory Th2 lymphocytes directly in atopic dermatitis, in addition to expanding the pool of systemic Th2 lymphocytes by migrating to the lymph nodes and directing Th0 cells toward Th2 differentiation.

Langerhans cells in patients with atopic dermatitis may also participate in the recruitment of $\mathrm{CD}^{+} \mathrm{T}$ lymphocytes to the lesion site by stimulating enhanced production of IL-16. Compared with inflammatory dendritic epidermal cells, relatively few plasmacytoid dendritic cells are present in atopic dermatitis lesions. This selective lack of plasmacytoid dendritic cells in atopic dermatitis lesions may be one of the reasons for the high susceptibility of patients with atopic dermatitis to viral skin infections. As mentioned previously, the majority of these patients are colonized with $S$. aureus, which is present both in lesional skin and, to a lesser extent, in nonlesional skin. Many factors likely contribute to this colonization, including an impaired epidermal barrier, an altered innate immune system, decreased bacterial clearance, and increased bacterial adhesion. It has also been shown that the presence of antimicrobial peptides, including $\beta$-defensins 2 and 3 , are significantly decreased in both acute and chronic lesions of atopic dermatitis. Moreover, decreased levels of dermcidin, a type of antimicrobial peptide with broad-spectrum activity that is constitutively expressed in eccrine sweat glands, secreted into sweat and transported to the epidermis, have been reported in atopic dermatitis lesions. ${ }^{36}$

\section{Psoriasis}

Psoriasis is one of the most frequent inflammatory skin disorders in Caucasians. It is a genetically determined disease that affects the skin, scalp, and nails. It is characterized by sharply demarcated erythematous plaques with silvery scales, which appear typically on the knees, elbows, sacral region, and scalp, but the entire skin may be involved. Histologic characteristics of psoriasis include abnormal epidermal proliferation and differentiation, as well as deviated activation of the immune system. Psoriatic arthritis occurs in 5\%-30\% of patients with cutaneous psoriasis and can appear in $10 \%-15 \%$ of patients before involvement of the skin. ${ }^{37,38}$ It can be manifested as mono- and asymmetric oligoarthritis, arthritis of the distal interphalangeal joints, rheumatoid arthritis-like changes, arthritis mutilans, and/or spondylitis and sacroiliitis.

Based upon analyses of family pedigrees, a polygenic inheritance provides the best model for the complex genetics of psoriasis. Linkage studies have demonstrated several genetic loci potentially responsible for development of psoriasis. ${ }^{37,38}$ PSORS1 has been mapped to chromosome $6 \mathrm{p} 21.3$, and is considered to be the major gene locus involving psoriasis. The critical region appears to be a $300 \mathrm{~kb}$ interval around the centromere of MHC Class I. Other gene loci linked to psoriasis include PSORS2. Several observations suggest that psoriasis is a $\mathrm{T}$ lymphocyte-mediated autoimmune disease. $\mathrm{T}$ lymphocytes already predominate 
in the cell infiltrate in psoriatic plaques in early lesions. $\mathrm{T}$ lymphocytes, including both $\mathrm{CD}^{+}$and $\mathrm{CD}^{+}$cells, are activated $\left(\mathrm{HLA}^{-} \mathrm{DR}^{+} / \mathrm{CD} 25^{+}\right)$. In the dermis, the $\mathrm{CD}^{+}$cells predominate, while $\mathrm{CD}^{+}$cells prevail in the epidermis. One of the earliest events in the psoriatic plaques is the influx of activated $\mathrm{CD}^{+}$cells. In resolving plaques, an influx of $\mathrm{CD}^{+}$cells predominates, while there is a concomitant decrease in $\mathrm{CD}^{+}$cells. $\mathrm{CD}^{+}$cells interact with dendritic cells expressing MHC Class II antigens, while $\mathrm{CD}^{+}$ cells interact with dendritic cells expressing MHC Class $\mathrm{I}$ antigens. The induction of $\mathrm{T}$ cell activation by psoriatic epidermal cells is highly dependent on the population of CD1a ${ }^{-} / \mathrm{HLA}^{-} \mathrm{DR}^{+}$dendritic cells, while CD1a $\mathrm{a}^{+}$Langerhans cells, HLA-DR ${ }^{+}$keratinocytes, and dermal dendrocytes might also be relevant antigen-presenting cells in psoriasis. Activated $\mathrm{T}$ lymphocytes produce two different patterns of cytokines, ie, Th1 cells which produce IL- 2 and IFN- $\gamma$ and Th2 cells which produce IL-4, IL-5, and IL-10. Psoriasis can be considered as a Th1 dominant disease. Activated T cells in the psoriatic plaques and other blood-derived cells have been shown to secrete a series of cytokines which may account for many characteristics of the psoriatic lesion. TNF$\alpha$, IL-3, IL-6, granulocyte-macrophage colony stimulating factor, and IFN- $\gamma$ are responsible for epidermal proliferation, TNF- $\alpha$ has been linked to the production of skin-associated antileukoproteinase and $\alpha$ - and $\beta$-defensins by epidermal cells, and to IL-8 for neutrophil accumulation. On the other hand, IL-10, which is secreted by Th2 lymphocytes, has been shown to inhibit the production of Th1 cytokines. ${ }^{37-39}$ The interaction between integrins of blood-derived cells and ICAM-1/VCAM-1 on endothelial cells of vessels in psoriatic plaques promotes cell migration to psoriatic plaques, and is crucial in the pathogenesis of psoriasis.

Dendritic cells present antigens to T lymphocytes, causing their activation. Activation of T lymphocytes is followed by clonal expansion of the population of T cells in psoriatic plaques. T lymphocyte receptor rearrangements suggest that specific antigen stimulation is relevant in the pathogenesis of psoriasis. ${ }^{37-39}$ Antigens activate lymphocytes in specific and nonspecific ways. Streptococcal superantigens may bind on chromosome 17q, PSORS3 on chromosome 4q, PSORS4 on chromosome 1q, PSORS5 on chromosome 3p, PSORS6 on chromosome 19p, and PSORS7 on chromosome 1p. The locus on chromosome 16q12-13, also known as PSORS8, has been shown to have linkage with both psoriasis and Crohn's disease. ${ }^{37-39}$ Superantigens activate T lymphocytes in a nonspecific way and induce the expression of cutaneous lymphocyte antigen on $\mathrm{T}$ lymphocytes.
Cutaneous lymphocyte antigen binds to E-selectin proteins on the endothelial cells, resulting in a preferential homing of T lymphocytes into the skin. In the process of activation, three sets of signals between Langerhans cells and T cells take place, ie, primary signals (eg, T cell receptor to $\mathrm{MHC}$ I or to MHC II), accessory signals (costimulation), and proliferation and differentiation signals. Costimulatory pathways between dendritic cells and the $\mathrm{T}$ lymphocyte are required for full T lymphocyte activation. One of these pathways is LFA-3 at the APC and CD2 on the surface of the T cell, resulting in $\mathrm{T}$ lymphocyte activation. Another costimulatory pathway is between CD80 and CD86 (the B7 molecule) on the APC and CD28 or CTLA4 on the T cell. CD28 transmits activation and CTLA4 inhibits T lymphocyte activity.

Clearly, the pathogenic cells in psoriasis are activated $\mathrm{T}$ lymphocytes. $\mathrm{T}$ cell activation requires interactions between a Langerhans cell and a T cell. Dendritic cells present a specific antigen to the $\mathrm{T}$ cell mediated through the $\mathrm{MHC}$ which associates with CD4 or CD8 on the T cell and ICAM-1 with LFA-1 (signal 1). The T lymphocyte recognizes this antigen through the $\mathrm{T}$ cell receptor (and signals through CD3). The costimulatory signal, referred to as signal 2, is accomplished via the dendritic cell and T cell interactions (CD80/CD86 with CD28 and LFA-3 with CD2). Biologic agents interact with either signal 1 or 2 and may block $\mathrm{T}$ cell activation. One of the representative drugs in this group is efalizumab (Raptiva $^{\circledR}$, anti-CD11a). It is a humanized antibody which binds to CD11a on T cells, which is a part of LFA-1, thus blocking the binding to ICAM-1 on dendritic cells. The LFA-1 to ICAM-1 interaction is also important for $\mathrm{T}$ cell adhesion to endothelial cells and prevents $\mathrm{T}$ cell migration into the inflamed skin. Clinical studies demonstrated improvement of the Psoriasis Area Severity Index in $62 \%$ of patients by $50 \%$, while $30 \%$ of patients had a $75 \%$ improvement. ${ }^{37-39}$

\section{Conclusion}

The importance of dendritic cells in infectious diseases and autoimmunity is dependent on the appropriate elicitation of the $\mathrm{T}$ cell response that is both beneficial to the host and also results in the elimination of the pathogen. Further research is needed to manipulate dendritic cell responses to pathogens, allergens, and selfantigens, leading to favorable $\mathrm{T}$ cell responses that would potentially lead to treatment of infectious diseases and autoimmune diseases.

\section{Acknowledgment}

This work was supported by Potts Memorial Foundation and startup funds from Western University of Health Sciences. 


\section{Disclosure}

The authors report no conflicts of interest in this work.

\section{References}

1. Clark GJ, Angel N, Kato M, et al. The role of dendritic cells in the innate immune system. Microbes Infect. 2010;2:257-272.

2. Steinman RM. Some interfaces of dendritic cell biology. APMIS. 2003; 111:675-697.

3. Colonna M. TLR pathways and IFN-regulatory factors: To each its own. Eur J Immunol. 2007;37:306-309.

4. Ardavín C, Martínez del Hoyo G, Martín P, et al. Origin and differentiation of dendritic cells. Trends Immunol. 2001;22:691-700.

5. Blom B, Ho S, Antonenko S, Liu YJ. Generation of interferon alpha-producing predendritic cell (Pre-DC)2 from human CD34(+) hematopoietic stem cells. J Exp Med. 2000;192:1785-1796.

6. Teleshova N, Kenney J, Robbiani M. Dendritic cells and HIV infection: Activating dendritic cells to boost immunity. Adv Dent Res. 2006;19: $36-41$.

7. Cavanagh LL, Von Andrian UH. Travellers in many guises: The origins and destinations of dendritic cells. Immunol Cell Biol. 2002;80: 448-462.

8. Manches O, Munn D, Fallahi A, et al. HIV-activated human plasmacytoid DCs induce Tregs through an indoleamine 2,3-dioxygenase-dependent mechanism. J Clin Invest. 2008;118:3431-3439.

9. Peterson JD, Herzenberg LA, Vasquez K, Waltenbaugh C. Glutathione levels in antigen-presenting cells modulate Th1 versus Th2 response patterns. Proct Natl Acad Sci U S A. 1998;95:3071-3076.

10. Piccioli D, Sbrana S, Melandri E, Valiante NM. Contact-dependent stimulation and inhibition of dendritic cells by natural killer cells. J Exp Med. 2002;195:335-341.

11. Marino S, Pawar S, Fuller CL, Reinhart TA, Flynn JL, Kirschner DE. Dendritic cell trafficking and antigen presentation in the human immune response to Mycobacterium tuberculosis. J Immunol. 2004;173: 494-506.

12. Melki MT, Saïdi H, Dufour A, Olivo-Marin JC, Gougeon ML. Escape of HIV-1-infected dendritic cells from TRAIL-mediated NK cell cytotoxicity during NK-Dc cross-talk-a pivotal role of Hmgb1. PLoS Pathog. 2010;6:e1000862.

13. Alter G, Kavanagh D, Rihn S, et al. IL-10 induces aberrant deletion of dendritic cells by natural killer cells in the context of HIV Infection. J Clin Invest. 2010;120:1905-1913.

14. Jegalian AG, Facchetti F, Jaffe EF. Plasmacytoid dendritic cells: Physiologic roles and pathologic states. Adv Anat Pathol. 2009;16:392-404.

15. Lehmann C, Lafferty M, Garzino-Demo AJ, et al. Plasmacytoid dendritic cells accumulate and secrete interferon alpha in lymph nodes of HIV-1 patients. PLoS One. 2010;5:e11110.

16. Kodama A, Tanaka R, Zhang LF, et al. Impairment of in vitro generation of monocyte-derived human dendritic cells by inactivated human immunodeficiency virus-1: Involvement of Type I interferon produced from plasmacytoid dendritc cells. Hum Immunol. 2010;71:541-550.

17. Geijtenbeek TB, van Duijnhoven GC, van Vliet SJ, et al. Identification of different binding sites in the dendritic cell-specific receptor Dc-sign for intercellular adhesion molecule 3 and HIV-1. J Biol Chem. 2002;277: 11314-11320.

18. Arrighi JF, Pion M, Garcia E, et al. Dc-sign-mediated infectious synapse formation enhances X4 Hiv-1 transmission from dendritic cells to T cells. J Exp Med. 2004;200:1279-1288.
19. Che KF, Sabado RL, Shankar EM, et al. HIV1 impairs in vitro priming of naive T Cells and gives rise to contact-dependent suppressor T cells. Eur J Immunol. 2010;40:2248-2258.

20. Venketaraman V, Rodgers T, Linnares R, et al. Tuberculosis immunity in healthy and HIV-infected subjects. AIDS Res Ther. 2006;3:5.

21. Herzenberg LA, de Rosa SC, Dubs JG, et al. Glutathione deficiency is associated with impaired survival in HIV disease. Proc Natl Acad Sci U S A. 1997;94:1967-1972.

22. Levy JA. HIV pathogenesis and long term survival. AIDS. 1993;7: 1401-1410.

23. Pantaleo G, Graziosi C, Fauci AS. New concepts in the immunopathogenesis of human immunodeficiency infection. N Engl J Med. 1993;328:327-335.

24. Eck HP, Gmunder H, Hartmann M, Petzoldt D, Daniel V, Droge W. Low concentrations of acid-soluble thiol (cysteine) in the blood plasma of HIV-1-infected patients. Biol Chem Hoppe Seyler. 1989;370: 101-108.

25. Korbel DS, Schneider BE, Schaible UE. Innate immunity in tuberculosis: Myths and truth. Microbes Infect. 2008;10:995-1004.

26. Chan J, Flynn J. The immunological aspects of latency in tuberculosis. Clin Immunol. 2004;110:2-12.

27. Rajashree P, Das SD. Infection with prevalent clinical strains of Mycobacterium tuberculosis leads to differential maturation of monocyte derived dendritic cells. Immunol Lett. 2008;117:174-180.

28. Spergel J. Epidemiology of atopic dermatitis and atop march in children. Immunol Allergy Clin North Am. 2010;30:269-280.

29. Capoluongo E, Giglio AA, Lavieri MM, et al. Genotypic and phenotypic characterization of Staphylococcus aureus strains isolated in subjects with atopic dermatitis. Higher prevalence of exfoliative B toxin production in lesional strains and correlation between the markers of disease intensity and colonization density. J Dermatol Sci. 2001;26:145-155.

30. Dworzak MN, Fröschl G, Printz D, et al. Skin-associated lymphocytes in the peripheral blood of patients with atopic dermatitis: Signs of subset expansion and stimulation. J Allergy Clin Immunol. 1999;103: 901-906.

31. Lebre MC, van Capel TM, Bos JD, Knol EF, Kapsenberg ML, de Jong EC. Aberrant function of peripheral blood myeloid and plasmacytoid dendritic cells in atopic dermatitis patients. J Allergy Clin Immunol. 2008;122:969-976.

32. Steinhoff M, Steinhoff A, Homey B, Luger TA, Schneider SW. Role of vasculature in atopic dermatitis. J Allergy Clin Immunol. 2006;118: 190-197.

33. Gros E, Bussmann C, Bieber T, Förster I, Novak N. Expression of chemokines and chemokine receptors in lesional and nonlesional upper skin of patients with atopic dermatitis. J Allergy Clin Immunol. 2009; 124:753-760.

34. Novak N, Koch S, Allam JP, Bieber T. Dendritic cells: Bridging innate and adaptive immunity in atopic dermatitis. J Allergy Clin Immunol. 2010;125:50-59.

35. Ong PY, Leung DYM. The infectious aspects of atopic dermatitis. Immunol Allergy Clin North Am. 2010;30:309-321.

36. Krueger JG. The immunologic basis for treatment of psoriasis with new biologic agents. J Am Acad Dermatol. 2002;46:1-23.

37. Singri P, West DP, Gordon KB. Biologic therapy for psoriasis. Arch Dermatol. 2002;138:657-663.

38. Gottlieb A, Miller B, Chaundhari U, et al. Clinical and histologic effects of subcutaneously administered anti-CD11a (hu1124) in patients with psoriasis. J Invest Dermatol. 2002;114:840.

International Journal of Interferon, Cytokine and Mediator Research

\section{Publish your work in this journal}

The International Journal of Interferon, Cytokine and Mediator Research is an international, peer-reviewed, open-access, online journal. The focus of the journal is to publish original research, reports, editorials, reviews and commentaries on all aspects of interferon, cytokine and mediators of inflammation from labora- tory science to therapeutic indications and clinical studies. The manuscript management system is completely online and includes a very quick and fair peer-review system, which is all easy to use. Visit http://www.dovepress.com/testimonials.php to read real quotes from published authors. 